\title{
Riscos e agravos na saúde dos profissionais de enfermagem no ambiente de trabalho
}

\author{
Jussara dos Santos Barreto*, Edvane Mauricio da Silva Rodrigues**, Diego Pereira Rodrigues***
}

\begin{abstract}
${ }^{*}$ Enfermeira do Hospital Estadual Azevedo Lima (HEAL), ${ }^{* *}$ Enfermeira do Laboratório Sérgio Franco Medicina Diagnóstica, Especialização em Enfermagem do Trabalho pela Universidade Gama Filho (UGF), Niterói RJ, ***Enfermeiro Supervisor de Campo do Centro Universitário Plinio Leite (UNIPLI), Especialização em Enfermagem do Trabalho pela Universidade Gama Filho (UGF), Niterói RJ
\end{abstract}

\section{Resumo}

O ofício de enfermagem no ambiente hospitalar, os acidentes e doenças ocupacionais estão relacionados a vários fatores de risco, principalmente às condiçóes inadequadas de trabalho. Buscou-se com o presente estudo identificar os riscos que estes profissionais estáo expostos na sua atividade laboral com materiais perfuro-cortantes e descrever os fatores causadores dos desajustes psicológicos dos profissionais de enfermagem. Trata-se de um estudo descritivo com abordagem metodológica qualitativa como método de revisão bibliográfica. A pesquisa foi realizada na Biblioteca Virtual de Saúde (BVS), tendo como bases de dados do estudo a BDENF e Scielo. A profissão de enfermagem é considerada de alto risco por conta de vários fatores que acarretam prejuízos a saúde do profissional, que ocorrem no seu cotidiano, principalmente por materiais perfuro-cortantes. A morte de crianças e adolescentes causa um desajuste nos profissionais de enfermagem, provocando problemas psicológicos e mentais que muitas das vezes provoca o seu afastamento (absenteísmo). Concluímos que o uso correto dos Equipamentos de Proteção Individual (EPIs) é essencial para a redução dos acidentes com materiais perfurocortantes. Consequentemente a instituição deve tomar medidas para a prevenção de riscos ocupacionais e problemas psicológicos de seus profissionais, tornando o ambiente de trabalho mais favorável e saudável para a saúde do trabalhador.

Palavras-chave: riscos ocupacionais, doenças profissionais, Enfermagem.

\section{Abstract}

\section{Risks and health problems of nursing professionals at work}

The profession of nursing in the hospital environment, accidents and diseases are related to several risk factors, especially inadequate working conditions. The aim of the present study was to identify the risks of work accidents caused by sharpedged material which these professionals are exposed to at work activities and to describe factors which cause psychological maladjustment of nursing professionals. This is a descriptive literature review with qualitative approach. The survey was conducted in the Virtual Health Library (VHL), with Scielo and BDENF databases. The nursing profession is considered high risk because of several factors that cause harm to health to the professional, during daily activities, particularly with sharp-edged material. The death of children and adolescents cause disturbance in professional nursing, causing mental and psychological 
problems that often causes absenteeism. We concluded that the correct use of Personal Protective Equipment (PPE) is essential to reduce accidents with sharp-edged material. Consequently, the institution should take measures to prevent risks of work accidents and psychological problems, and to promote employee's health and maintain a pleasant health environment.

Key-words: occupational hazards, diseases, nursing.

\section{Resumen}

\section{Riesgos y daños a la salud de profesionales de enfermería en el trabajo}

En el oficio de enfermería en el ámbito hospitalario, los accidentes y las enfermedades están relacionados con varios factores de riesgo, especialmente en condiciones de trabajo inadecuadas. Hemos tratado de identificar con el presente estudio el riesgo a que estos profesionales están expuestos en sus actividades de trabajo con materiales corto-punzantes y describir los factores causantes de desajuste psicológico de los profesionales de enfermería. Es un estudio descriptivo con enfoque metodológico cualitativo como método de revisión de la literatura. La investigación se realizó en la Biblioteca Virtual en Salud (BVS), utilizando las bases de datos Scielo y BDENF. La profesión de enfermería se considera de alto riesgo debido a varios factores que causan daño a los profesionales de la salud que ocurren en su cotidiano, especialmente con materiales corto-punzantes. La muerte de niños y adolescentes provoca un desajuste en los profesionales de enfermería, causando problemas mentales y psicológicos que a menudo provoca absentismo. Se concluyó que el uso correcto de Equipos de Protección Individual (EPI) es esencial para reducir los accidentes con materiales corto-punzantes. En consecuencia, la institución debe tomar medidas para la prevención de riesgos y problemas psicológicos de los profesionales y proporcionar al trabajador un ambiente de trabajo más favorable y saludable.

Palabras-clave: riesgos laborales, las enfermedades, de enfermería.

\section{Introdução}

A profissão de enfermagem dentro de suas atribuiçóes realiza vários procedimentos invasivos, principalmente, a administração de medicamentos, o que torna o profissional mais susceptível à ocorrência de acidentes. Necessitando o profissional de esclarecimentos e orientaçóes sobre as doenças ocupacionais e estabelecendo um nexo causal com a sua atividade exercida.

Essas doenças ocupacionais acarretam o afastamento do profissional do trabalho, prejudicando a saúde física e psicológica do trabalhador. Tais agravos fazem com que profissionais fiquem expostos a uma série de fatores de risco, comprometendo, assim, a sua saúde.

A motivação para realizar o presente estudo decorreu da atuação de experiências profissionais de enfermagem em um hospital de médio porte, na região metropolitana do Estado do Rio de Janeiro. É importante ressaltar que esses trabalhadores sempre relatavam estar estressados, sobrecarregados, e, além disso, faltavam recursos materiais e humanos, instabilidade no emprego, baixos salários, desvalorização pessoal e profissional por parte da gerência administrativa e operacional, sendo essas questóes determinantes para destabilizar o profissional e ocasionar $o$ acidente.
Vários estudos destacam que os hospitais são entidades normalmente associadas à prestação de serviços à saúde, visando à assistência, à prevenção, à terapêutica e à cura daqueles acometidos pela doença. Porém, também podem ser responsáveis pela ocorrência de uma série de riscos à saúde daqueles que trabalham na instituição, tais como os acidentes de trabalho, as doenças profissionais e as doenças de trabalho. Os acidentes de trabalho representam grande importância e complexidade na saúde ocupacional, seja de origem, física, biológica, química ou ergonômica. Devemos considerar que a problemática dos riscos assume maiores proporçóes do que as estatísticas conseguem estimar. Sendo que a sua real dimensão é dificultada por diversos fatores, principalmente a subnotificaçáo de acidentes de trabalho [1].

A evolução silenciosa e demorada das doenças ocupacionais dificulta a percepçáo do nexo causal entre o trabalho e o agravo, além do despreparo e a falta de informação dos profissionais de saúde em reconhecer e relacionar as atividades laborais aos riscos ocupacionais aos que estão expostos [1].

Para entendermos melhor a questão, é necessário reconhecer que risco à saúde ocupacional é toda e qualquer ocorrência gerada no trabalho que não seja seguro. A etapa de antecipação dos riscos é 
fundamental, pois envolve a análise de projetos de novas instalações, métodos ou processos de trabalho, ou de modificação dos já existentes. Visando, assim, a identificação dos riscos potenciais e reais na saúde do trabalhador e implementando medidas de proteção para a redução ou eliminação. Deve-se lembrar que um ambiente de trabalho seguro e saudável protege os trabalhadores de ameaças à sua saúde e ao mesmo tempo amplia a capacidade para o trabalho e a produtividade [2].

Há ocasióes em que os riscos são identificados após o comprometimento da saúde do trabalhador. Trata-se, então, de uma situação paradoxal, pois ao mesmo tempo em que o hospital tem como missão de salvar vidas e recuperar a saúde dos clientes, favorece o adoecimento de trabalhadores, devido à elevada jornada de trabalho, baixa remuneração, tensão emocional advinda do cuidado direto de pessoas fisicamente doentes, ocasionando danos à saúde dos profissionais [3].

Diante desta problematização, o objeto de estudo pretende identificar quais os fatores que contribuem para os riscos da saúde dos profissionais de enfermagem. Nessa perspectiva os objetivos do estudo pretendem identificar os fatores de riscos a que os profissionais de enfermagem estáo expostos na sua atividade laboral com materiais perfurocortantes e descrever os fatores causadores dos desajustes psicológicos dos profissionais de enfermagem.

Salientamos que a justificativa deste estudo é alertar os profissionais de saúde, especificamente os de enfermagem, sobre os riscos pertinentes ao seu ambiente de trabalho, advindos da falta de conscientização e orientaçóes acerca de seu cotidiano, principalmente no que tange aos acidentes de trabalho, ocasionando um grande número de profissionais acidentados.

Nesse sentido, pontua que os profissionais de enfermagem vivenciam situaçóes de risco cotidianamente, deixando de proteger-se, de cuidar-se, como se fosse uma atitude "natural", essencial para o exercício da profissão, cujo objeto é a prática do cuidar. Observa-se que, muitas vezes, a atenção da equipe no ambiente de trabalho se concentra no cuidar, porém, no cuidar apenas dos outros [4].

Acredita-se que este estudo possa contribuir de forma precisa e sistematizada no cotidiano dos trabalhadores da saúde, principalmente os profissionais de enfermagem, como também propiciar reflexôes acadêmicas sobre a temática, aumentando o acervo bibliográfico para o conhecimento em questão.

\section{Material e métodos}

Trata-se de uma pesquisa descritiva com abordagem qualitativa. $\mathrm{Na}$ abordagem qualitativa, os estudos buscam a realidade que não pode ser quantificada, o pesquisador observa os fatos sob a ótica de alguém buscando compreensão do contexto [5]. Como método descritivo entende-se, aqui, que descrever é narrar o que acontece e, desta forma, mantivemo-nos estritamente dentro do declarado pelos entrevistados e de suas descriçóes dos fatos e problemas por eles vivenciados [6].

Visando atender aos objetivos especificados neste artigo, utilizou-se o método de revisão de literatura, que tem como finalidade sintetizar resultados obtidos em pesquisas relativos ao tema ou a uma questão específica, de forma sistemática e ordenada com o intuito de contribuir para o conhecimento dessa temática [7].

O levantamento bibliográfico foi realizado em setembro de 2010, com base na Biblioteca Virtual de Saúde (BVS), cujas bases de dados utilizadas foram BDENF e Scielo.

Para a coleta de dados utilizamos os seguintes descritores: Riscos ocupacionais, doenças profissionais, enfermagem. Destaca-se que trabalhamos com todo tipo de bibliografia encontrada na BVS, ou seja, artigo, dissertação, monografia, dentre outras; assim como toda produção temporal entre no período de 2001 a 2011. Ressalta-se que, inicialmente, pesquisamos cada descritor individualmente, conforme o Quadro 1 abaixo.

Quadro 1 - Distribuição quantitativa das bibliografias encontradas nas bases de dados BDENF e Scielo.

\begin{tabular}{|l|c|c|c|}
\hline \multicolumn{1}{|c|}{ Descritores } & \multicolumn{3}{c|}{ Banco de Dados: BVS } \\
\hline & BDENF & Scielo & Total \\
\hline Riscos ocupacionais & 262 & 84 & 366 \\
\hline Doenças profissionais & 267 & 314 & 581 \\
\hline Enfermagem & 14.128 & 3.383 & 17.511 \\
\hline Total & 14.657 & 3.781 & 18.438 \\
\hline
\end{tabular}

Após a coleta inicial, percebemos que seria necessário um refinamento pelo número excessivo de resultados encontrados. Desta forma, optamos por realizar uma nova busca com os descritores em associação em dupla, conforme o Quadro 2. 
Quadro 2 - Distribuição quantitativa das bibliografias encontradas nas bases de dados Scielo e BDENF para análise dos dados, conforme os descritores associados em dupla.

\begin{tabular}{|l|l|l|l|}
\hline \multicolumn{1}{|c|}{ Descritores } & BDENF & Scielo & Total \\
\hline $\begin{array}{l}\text { Riscos ocupacionais + Doen- } \\
\text { ças profissionais }\end{array}$ & 36 & 4 & 40 \\
\hline $\begin{array}{l}\text { Riscos ocupacionais + Enfer- } \\
\text { magem }\end{array}$ & 184 & 20 & 204 \\
\hline $\begin{array}{l}\text { Doenças profissionais + } \\
\text { Enfermagem }\end{array}$ & 177 & 30 & 207 \\
\hline Total & 397 & 54 & 451 \\
\hline
\end{tabular}

Ressalta-se que, ao realizarmos a busca em trio dos descritores, não foram encontradas referências bibliográficas suficientes, apenas 01 referência na base de dados Scielo para a realização das leituras. Então realizamos a pré-leitura, cujo objetivo é ter uma visão global do assunto apresentado e, assim, refinar os artigos encontrados [6]. Para encontrar a seleção da bibliografia potencial, realizamos uma leitura seletiva, crítica e interpretativa de todas as pesquisas, cujos dados, após a análise foram categorizados de forma a responderem ao problema da pesquisa. Destaca-se que dentre os artigos selecionados como potenciais houve o descarte de 445 por não se enquadrar no objeto de pesquisa. Assim chegamos a um total de 06 artigos potenciais para análise de dados, conforme o Quadro 3.

\section{Resultados e discussão}

\section{Os riscos pertinentes à profissáo de enfermagem}

Os profissionais de enfermagem estão submetidos a diversos riscos pertinentes em sua rotina de atividades, como manuseio de materiais perfurocortantes, que é considerado o principal causador de acidentes de trabalho no âmbito hospitalar por essa categoria de saúde.

No cotidiano do trabalho da equipe de enfermagem é comum observar-se situaçóes de riscos a saúde ocupacional, tais como: administração de banhos nos clientes com ausência de EPI apropriados, realização de coleta de sangue e outros líquidos corpóreos veiculadores de microrganismos patogênicos, administraçáo de medicamento e o seu descarte apropriado, que geralmente estão localizadas longe dos locais das coletas ou da administração, e ainda com a capacidade esgotada em muitas das vezes. Além de o uso de calçados abertos e portando adornos o que facilitam a possibilidade de contaminaçáo, ocasionando riscos ao trabalhador e aos seus colegas no ambiente de trabalho [8].

A Enfermagem é considerada uma profissão de alto risco, isso se deve principalmente pela falta de esclarecimento sobre as medidas de segurança, condiçôes inadequadas e insalubres no ambiente de trabalho, sobrecarga de atividades laborais, risco de contrair patologias e de se acidentar a qualquer momento. Sendo considerado por esse grupo como fator determinante para acidente com material perfurocortantes [9]. É importante ressaltar que o profissional deve obter o esclarecimento ou as devidas informações sobre as medidas de proteção, como o uso correto dos Equipamentos de Proteção Individual (EPI) e as medidas de biossegurança. Devendo ser transmitidas essas informações pelos seus superiores altamente qualificados ou por conta de algum setor especializado nessa temática, como, por exemplo, a Comissão de Controle de Infecçóes

Quadro 3 - Distribuição das bibliografias potenciais para a construção da pesquisa.

\begin{tabular}{|l|l|l|l|l|}
\hline \multicolumn{1}{|c|}{ Autor (ES) } & Ano & \multicolumn{1}{c|}{ Título } & \multicolumn{1}{c|}{$\begin{array}{c}\text { Base de } \\
\text { dados }\end{array}$} & $\begin{array}{c}\text { Tipo de } \\
\text { publicação }\end{array}$ \\
\hline $\begin{array}{l}\text { Robazzi MLCC et } \\
\text { al. [8] }\end{array}$ & 2004 & $\begin{array}{l}\text { A norma regulamentadora 32 e suas implicações sobre os } \\
\text { trabalhadores de enfermagem. }\end{array}$ & Scielo \\
\hline $\begin{array}{l}\text { Alves SSM et al. } \\
\text { [9] }\end{array}$ & 2009 & $\begin{array}{l}\text { Acidentes com perfurocortantes em trabalhadores de enferma- } \\
\text { gem uma questão de biossegurança. }\end{array}$ & BDENF & Artigo \\
\hline $\begin{array}{l}\text { Simão SAF et al. } \\
\text { [10] }\end{array}$ & 2010 & $\begin{array}{l}\text { Acidentes de Trabalho com material Perfurocortante Envolven- } \\
\text { do Profissionais de Enfermagem na Unidade de Emergência } \\
\text { Hospitalar }\end{array}$ & BDENF & Artigo \\
\hline $\begin{array}{l}\text { Silvia MKD et al. } \\
\text { [11] }\end{array}$ & 2009 & $\begin{array}{l}\text { Riscos ocupacionais em um setor de hemodiálise na perspectiva } \\
\text { dos trabalhadores da equipe de enfermagem. }\end{array}$ & BDENF & Artigo \\
\hline $\begin{array}{l}\text { Martins JT et al. } \\
\text { [12] }\end{array}$ & 2009 & $\begin{array}{l}\text { O trabalho do enfermeiro em unidade de terapia intensiva: } \\
\text { sentimento de sofrimento. }\end{array}$ & BDENF & Artigo \\
\hline $\begin{array}{l}\text { Martinato MCNB } \\
\text { et al. [13] }\end{array}$ & 2010 & Absenteísmo na enfermagem: uma revisão integrativa. & SciELO & Artigo \\
\hline
\end{tabular}


Hospitalares $(\mathrm{CCIH})$, onde o trabalhador deve ser submetido a um treinamento teórico e prático adequado.

A Norma Regulamentadora (NR-32) estabelece diretrizes básicas para a implementaçáo de medidas de proteção à segurança e à saúde dos trabalhadores em estabelecimentos de assistência à saúde, em todos os níveis de complexidade, em regime de internação [14].

Desse modo, outro fator importante está relacionado ao termo biossegurança que tem assumido, através da Lei n. 8.975 de 05 de janeiro de 1995 , que criou a Comissão Técnica Nacional de Biossegurança, uma dimensão ampla, que extrapola a área da saúde e do trabalho, sendo empregada quando há referência ao meio ambiente e à biotecnologia [15] .

Assim, na prevenção de acidentes no ambiente do trabalho, os esforços devem ser concentrados inicialmente na eliminação dos perigos e dos riscos, não permitindo interação direta entre pessoas e perigo e, posteriormente, orientaçóes, treinamento e fornecimento de EPI [16].

Com a combinação dessas medidas, é possível obter melhores resultados na prevenção de acidentes do trabalho, de doenças ocupacionais e de doenças profissionais, as quais a grande maioria dos acidentes de trabalho em hospitais ocorre com os profissionais de enfermagem, ocasionando muitas vezes, o seu afastamento e elevando os índices de absenteísmo na instituição, devido principalmente a ausência ou uso incorreto de EPI.

Os EPI devem ser fornecidos obrigatoriamente pelo empregador, devendo orientar o trabalhador pelo uso correto nas suas atividades laborais e também a sua conservação, conforme a Norma Regulamentadora (NR-6), como o uso de máscaras, gorro, óculos, capote, botas, entre outros, tornando-se obrigatório a todos os trabalhadores. O comprometimento da adequada realização destas medidas proporciona maior proteçáo contra agravos à saúde ocupacional.

É relevante lembrar, que os altos índices de acidentes com objetos perfurocortantes no setor hospitalar se somam ao significante contingente de profissionais sem a devida proteção vacinal. Altamente agravante, por conta de acidentes com materiais biológicos, uma vez que são causadores de muitos problemas de saúde dos trabalhadores $[17,18]$.

A manipulação de agulha (reencape) é o maior risco de acidente por material penetrante entre trabalhadores hospitalares, e também o descarte inadequado de objetos perfurocortantes. $\mathrm{E}$, em relação à utilização dos EPI no momento do acidente, a maioria dos profissionais não dá a real importância do seu uso para a prevenção dos acidentes de trabalho $[17,18]$.

Vale ressaltar que o setor de saúde ocupacional na instituição deve ter um controle do esquema vacinal (doses e reforços vacinais) de todos os profissionais envolvidos, seja efetivado, contratado, cooperativado ou terceirizado, contribuindo com a diminuição da contaminação de agravos imunopreveníveis. E também institucionalizar protocolos de medidas de biossegurança para evitar os principais acidentes com objetos perfurocortantes como o reencape de agulhas e o descarte inadequado desses objetos, embora o uso da proteção individual não impeça que o trabalhador sofra o acidente, reduz o seu risco.

Uma vez que o trabalhador teve contato acidental, certo ou duvidoso com materiais biológicos humanos, o mesmo deverá ser submetido pelo menos à sorologia para SIDA/AIDS (anti-HIV), Hepatite B (anti-HBC total) e Hepatite C (anti-HCV) nos tempos zero (data acidental), três e seis meses após o acidente [19].

\section{Aspectos institucionais resguardando a saúde e a qualidade de vida do trabalhador}

Os riscos existentes no ambiente do trabalho no exercício dos profissionais de enfermagem são resguardados perante órgãos institucionais, que utilizam açôes para o controle, diminuição e prevenção dos acidentes. Podemos observar também, que o trabalhador é resguardado perante os seus direitos trabalhistas e previdenciários caso ocorram acidentes de maior proporçáo e responsabilidade, impedindo, assim, o profissional de executar as suas atividades.

Diante desta problemática, devem-se buscar estratégias que possam contribuir para a prevenção dos acidentes de trabalho e promoção à saúde do trabalhador. Estratégias essas que devem ser institucionalizadas e trabalhadas com o fortalecimento das Comissóes Internas de Prevençóes de Acidentes (CIPA), assim como todas as demais estruturas organizacionais que se encarregam da educação [20].

Os meios e as alternativas que as instituiçóes devem proporcionar aos trabalhadores são essenciais, favorecendo a diminuição, redução e prevenção de acidentes e doenças profissionais. Devendo esses 
órgãos institucionais com as Comissões Internas de Prevençóes de Acidentes e Comissões de Controle de Infecçóes Hospitalares programarem medidas de controle e prevenção dos acidentes, melhorando o ambiente do trabalho e favorecendo a qualidade de vida do trabalhador.

Devemos destacar, que a Lei no 8.213, de 24 de julho de 1991, alterada pelo Decreto no 611, de 21 de julho de 1992 , no artigo $19^{\circ}$, reiterada ainda pela Lei Estadual do Paraná no 10.692 de 27 de dezembro de 1993, acidente do trabalho é aquele que ocorre pelo exercício do trabalho, a serviço da empresa ou, ainda, pelo serviço de trabalho de segurados especiais, provocando lesáo corporal ou perturbação funcional que cause a morte, a perda ou redução da capacidade para o trabalho, permanente ou temporária [17].

Dessa forma, a Comunicação de Acidente de Trabalho (CAT) é um instrumento extremamente importante na análise epidemiológica dos acidentes, pelas informaçóes que pode disponibilizar, porém se torna limitada quando o registro dos dados não é feito de forma correta ou as anotaçôes são incompletas. Muitas vezes os acidentes de trabalho não são notificados, como também não há seguimento adequado dos funcionários acometidos [16].

A comunicação de acidente de trabalho foi prevista inicialmente na Lei no 5.316/67 com todas as alteraçóes ocorridas posteriormente até a Lei ${ }^{\circ}$ 9.032/95, regulamentada pelo decreto $\mathrm{n}^{\circ} 2.172 / 97$. A Lei $n^{\circ} 8.213 / 91$ determina no seu artigo 22 que todo acidente do trabalho ou doença profissional deverá ser comunicado pela empresa ao INSS nas primeiras 24 horas após o acidente, sob pena de multa em caso de omissão [17].

É importante ressaltar que o preenchimento correto da comunicação de acidente de trabalho é ideal, pois permite verificar os principais tipos de acidentes na instituição e planejar as medidas de controle e prevenção dos acidentes. O profissional sempre deve requerer da empresa a sua via de preenchimento resguardando os seus direitos perante o acidente de trabalho, e das seis vias preenchidas, enviar uma via a cada órgão, conforme a descrição: Instituto Nacional de Seguridade Social (INSS), instituiçáo, trabalhador ou dependente, sindicato de classe do trabalhador, Sistema Único de Saúde (SUS) e a Delegacia Regional do Trabalho (DRT).

A comunicação do acidente do trabalho é primordial, pois essa documentação resguarda todos os direitos legais, trabalhistas e previdenciários do trabalhador, sendo importante que a instituição faça valer os aspectos legais a que a comunicação se insere e impedindo que o trabalhador tenha qualquer problema futuro. É necessário que as instituiçóes evitem o descaso sobre os acidentes, pois só comunicam em caso de maiores proporçóes na saúde e qualidade de vida do trabalhador, esquecendo que esse acidente pode aparecer seus danos na saúde do trabalhador mais tarde.

É importante lembrar, que nesse contexto de acidentes com material perfurocortantes, que o trabalhador acidentado por material biológico torna-se reagente em três ou seis meses, deve o médico responsável notificar novamente o INSS, para avaliar entáo, o tipo de marcador sorológico reagente, baseado nos conhecimentos científicos atuais sobre as doenças pesquisadas.

Assim, o trabalhador com uma doença ocupacional tem o direito a pedir afastamento de suas atividades por motivo de doença. O Instituto Nacional de Seguridade Social irá conceder o trabalhador o auxílio-doença, necessitando comprovar a doença relacionada ao seu emprego atual e, além disso, deve ter um mínimo de 12 meses de contribuição ao INSS $[12,18]$.

\section{$O$ vínculo enfermeiro-cliente e seus problemas psíquicos e psicológicos no ambiente do trabalho}

Esta categoria aborda os sofrimentos psíquicos, psicológicos e mentais nos trabalhadores da enfermagem causando desajustes aos mesmos por conta do processo de cuidado de cliente com risco de morte.

O processo da morte é algo natural, mas não é visto, conforme as perspectivas dos trabalhadores de enfermagem. A morte é mais bem aceita quando se tem o sentimento de que a pessoa já cumpriu as etapas da vida, entretanto, de acordo com os fatos da morte, o profissional de enfermagem tem a dificuldade encarar e aceitar quando se trata de crianças e adolescentes, pois são situaçóes que não ocorrem no ciclo natural da vida, e, muitas vezes, desajusta o seu psicológico e emocional, ocasionando dificuldade na execução de suas atividades [13].

Muitas vezes, o vínculo com os clientes pode estar relacionado ao tempo de internação na unidade, quando permanecem internados por um longo período, proporcionando a aproximação com a equipe de enfermagem. Dessa forma, os trabalhadores correm o risco de projetar o sofrimento em 
si mesmo, misturando os seus sentimentos, pois os vínculos estabelecidos estáo relacionados ao aspecto humanizado da enfermagem, fazendo com que aflore a necessidade de dedicaçáo, envolvimento, gerando sentimentos de sofrimento diante da fragilidade do outro [13].

Desse modo, a equipe de enfermagem fica fragilizada muitas das vezes, quando implementa o seu cuidado terapêutico se deparando com clientes em situaçóes graves a saúde, o que causa sofrimento no profissional. Assim, esse desajuste causado na equipe de enfermagem pode afetar profundamente o trabalhador, por conta do vínculo estabelecido, devido a fatos anteriores ou pessoais que o aproximam novamente da dramática em questáo e vivenciam outras situaçóes em que novamente ficam estagnados perante o seu cuidado, causando um sofrimento mental e psicológico, que poderá ocasionar o seu afastamento de suas atividades.

\section{Problemas psicológicos e sua relaçáo com o ab- senteísmo}

Esta categoria aborda a relação do absenteísmo, constituindo o afastamento do profissional de suas atividades com os sofrimentos psíquicos, psicológicos nos trabalhadores da enfermagem, interferindo diretamente na qualidade da saúde do trabalhador.

A doença ou agravo tem como significado a falta ou a perturbação da saúde, e o absenteísmo é a ausência de trabalhadores ao trabalho por motivo de acidente ou doença profissional. O profissional da enfermagem precisa lidar com a angústia do sofrimento dos clientes assistidos no seu trabalho, os quais encontram o processo de morte. Deste modo, os riscos mentais ocasionados por pressóes psicológicas no cuidado com o cliente assistido é altamente relevante, ocasionando um problema no trabalhador por conta de um desajuste psicológico e mental do profissional de enfermagem [13].

A profissão da enfermagem é uma das mais susceptíveis aos acidentes no ambiente do trabalho e doenças profissionais, isso ocorre porque suas atividades estáo direcionadas ao fazer da saúde, um prestar de assistência direta ao cliente, assim, possuindo uma ligaçáo direta ao absenteísmo [13].

A instituição deve utilizar instrumentos apropriados para prevenir e diminuir os índices de absenteísmo na profissão de enfermagem. Tornando as condições de trabalho mais adequadas e saudáveis, para minimizar os efeitos causados pelo absente- ísmo, verificando um suporte psicológico, com profissionais qualificados dentro da instituiçáo para os trabalhadores desajustados psicologicamente, e enfatizar o relacionamento interpessoal e a divisão adequada das atividades de enfermagem perante aos seus trabalhadores.

\section{Conclusão}

Os trabalhadores de enfermagem se submetem às relaçóes, às condiçóes e aos ambientes de trabalho geradores de insalubridade, colocando-se diante de riscos ocupacionais diversos que favorecem ao aparecimento de acidentes, sofrimentos e doenças.

Seguindo essa linha de pensamento, a profissáo enfermagem é considerada de alto risco para os seus trabalhadores, e isto se deve a diversas situações em que a equipe de enfermagem está submetida ao risco ocupacional. Dentre os principais causadores de acidentes biológicos encontramos os materiais perfurocortantes. Para reduzi-los, os profissionais devem receber por parte dos empregadores a devida orientação sobre o uso adequado dos EPI, além de garantir açóes para a prevenção e diminuição dos acidentes perfurocortantes.

Além dos riscos ocupacionais, há também a limitação dos profissionais de enfermagem, devido a problemas emocionais, psicológicos e mentais, que causa, na maioria das vezes, o afastamento do profissional de suas atividades, levando ao absenteísmo e prejudicando diretamente a qualidade de vida do trabalhador. Assim, deve a instituição tomar as melhores medidas para a prevenção e diminuição dos índices de absenteísmo, tornando o ambiente de trabalho mais favorável e saudável para a saúde do trabalhador.

\section{Referências}

1. Rapparini C, Cardo DM, Principais doenças infecciosas diagnosticadas em profissionais de saúde. In: Mastroeni MF. Biossegurança aplicada a laboratórios e serviços de saúde. São Paulo: Atheneu; 2004.

2. Reinhardt EL, Fischer FM. Barreiras às intervençóes relacionadas à saúde do trabalhador do setor saúde no Brasil. Rev Panam Salud Pública 2009;25(5):411-7.

3. Pitta A. Hospital: dor e morte como ofício. 5 a ed. São Paulo: Annablume; 2003.

4. Souza M. Acidentes ocupacionais e situaçóes de risco para equipes de enfermagem: um estudo em cinco hospitais do município de São Paulo [Dissertação]. São Paulo: Universidade de São Paulo; 1999.

5. Demo P. Educar pela pesquisa. Campinas: Autores Associados; 1997. 
6. Silva JLL, Souza SL. Fatores de risco para hipertensão arterial sistêmica versus estilo de vida docente. Revista Eletrônica de Enfermagem 2004;6(3):330-5.

7. Roman AR, Friedlander MR. Revisão integrativa de pesquisa aplicada à enfermagem. Cogitare Enferm1998;3(2):109-12.

8. Robazzi MLCC, Marziale MHP. A norma regulamentadora 32 e suas implicaçóes sobre os trabalhadores de enfermagem. Rev Latinoam Enfermagem 2004;12(5):834-36.

9. Alves SSM, Passos JP, Tocantins FR. Acidentes com perfurocortantes em trabalhadores de enfermagem: uma questâo de biossegurança. Rev Enfermagem UERJ 2009;17(3):373-77.

10. Simão SAF, Soares CRG, Souza V, Borges RAA, Cortez EA. Acidentes de trabalho com material perfurocortante envolvendo profissionais de enfermagem de unidade de emergência hospitalar. Rev Enfermagem UERJ 2010;18(3):400-4.

11. Silva MKD, Zeitoune RCG. Riscos ocupacionais em um setor de hemodiálise na perspectiva dos trabalhadores da equipe de enfermagem. Esc Anna Nery Rev Enfermagem 2009;13(2):279-86.

12. Martins JT, Robazzi MLCC. O trabalho da enfermagem em unidade de terapia intensiva: sentimentos de sofrimento. Rev Latinoam Enfermagem 2009;17(1):52-8.
13. Martinato MCNB, Severo DF, Marchand EAA, Siqueira $\mathrm{CH}$. Absenteísmo na Enfermagem: uma revisão integrada. Revista Gauch Enferm 2010;31(1):160-6.

14. Equipe Atlas. Segurança e Medicina do Trabalho. 67a ed. São Paulo: Atlas; 2011.

15. Caixeta RB, Branco AB. Acidente de trabalho com material biológico, em profissionais de saúde de hospitais públicos do Distrito Federal. Cad Saúde Pública 2005;21(3):737-46.

16. Ruiz MT, Barboza DB, Soler ZASG. Acidentes de trabalho: um estudo sobre esta ocorrência em um hospital geral. Arq Ciênc Saúde 2004;11(4):219-24.

17. Filemes STR. Acidentes com profissional de enfermagem no ambiente hospitalar: medidas de prevenção [Dissertação]. Rio de Janeiro: Centro Universitário Plínio Leite; 2009.

18. Brasil. Ministério da Saúde. Secretaria de Assistência a Saúde. Segurança no ambiente hospitalar. Brasília: Ministério da Saúde; 1995.

19. Rouquayrol MZ, Naomar AF. Epidemiologia \& Saúde. 5a ed. Rio de Janeiro: Medsi; 1999.

20. Deslandes SF. Análise do discurso oficial sobre a humanização da assistência hospitalar. Ciênc Saúde Coletiva 2004;9(1):7-14. 